\title{
Antifibrose-Wirkstoff bremst auch Husten bei IPF
}

Hintergrund u. Fragestellung: Husten ist ein typisches, oft sehr belastendes Symptom der Idiopathischen Pulmonalen Fibrose (IPF). Effektive Therapien des IPF-assoziierte Hustens fehlen, die üblichen Antitussiva wirken meist nicht. In dieser multizentrischen, prospektiven, einarmigen Beobachtungsstudie wurde der Effekt von Pirfenidon auf Husten bei IPF-Patienten geprüft.

\section{Originalie}

van Manen MJG, Birring SS, Vancheri $C$ et al. Effect of pirfenidone on cough in patients with idiopathic pulmonary fibrosis. Eur Respir J. 2017, 19;50(4). doi:10.1183/13993003.01157-2017
Patienten/Methoden: Einbezogen in die Studie waren 43 IPF-Patienten (FVC $\geq 50 \%$ Soll, DLCO $\geq 30 \%$ Soll) mit IPF-bezogenem Husten für $\geq$ 8 Wochen und Hustenscore von $\geq 40 \mathrm{~mm}$ auf der $0-100$ mm visuellen Analogskala (VAS). Sie erhielten für 12 Wochen Pirfenidon, ihre Hus- tenfrequenz wurde mit dem Leicester Hustenmonitor (LCM) vor der Therapie und 4 bzw. 12 Wochen nach Therapiestart erfasst.

Ergebnisse: 31 Patienten haben die Studie beendet. Die 12-wöchige Behandlung mit Pirfenidon führte zu einer Reduktion der Hustenfrequenz gemessen mit dem LCM um $34 \%$. Eine Verbesserung war bei 20 von 27 (74 \%) der Patienten nachzuweisen. Am Tag wie nachts waren vergleichbare Effekte erkennbar. Auch subjektive Methoden zeigten eine Abnahme des Hustens durch die Pirfenidon-Gabe (LCQ - Leicester Cough Questionnaire; VAS - cough und urge to cough). Bereits nach 4-wöchiger Behandlung ließ sich ein - wenn auch geringerer, aber signifikanter - Effekt auf die Hustenhäufigkeit durch Pirfenidon nachweisen (14\% Reduktion; Verbesserung bei 24/35 Patienten (69\%)).

Schlussfolgerung: Pirfenidon verringert die Hustenfrequenz bei IPF-Patienten.

\section{- Kommentar von Prof. Dr. med. Philipp Markart und Prof. Dr. med. Andreas Günther \\ Die Studie hat Limationen, die Daten sind aber vielversprechend}

Husten trägt sehr zur Einschränkung der Lebensqualität bei IPF-Patienten bei und ist mit einer schlechten Prognose assoziiert [1]. Die Pathogenese des Hustens ist hier nur unzureichend verstanden. Der IPF-bezogene Husten ist meist therapierefraktär, klassische Antitussiva sind nicht wirksam. Bisher gibt es nur sehr wenige Studien, in denen Therapien geprüft wurden: In einer randomisierten Studie konnte gezeigt werden, dass sich Thalidomid positiv auf die hustenspezifische Lebensqualität bei IPF-Patienten auswirkt. Eine objektive Messung der Hustenfrequenz erfolgte hier nicht, die Patientenzahl war klein und es traten häufig (74 \%) Nebenwirkungen auf [2]. In einer weiteren randomisierten, doppelblinden, placebokontrollierten Phase-2-Studie wurde gezeigt, dass inhalative Cromoglicinsäure bei IPF die Hustenfrequenz reduziert, nicht jedoch bei Patienten mit chronisch idiopathischem Husten [3].

Die vorliegende Studie untersuchte nun erstmals den Effekt eines bei IPF zugelassenen antifibrotischen Medikamentes auf den IPF-bezogenen Husten. Mittels objektiver und subjektiver Methoden konnte ein positiver Effekt auf die Hustenfrequenz, sowohl tagsüber wie auch nachts, durch die Behandlung mit Pirfenidon nachgewiesen werden.

Insgesamt sind die Daten vielversprechend, die Studie hat jedoch Limitationen. Zunächst: Die Patientenzahl ist klein und die Studie ist nicht placebokontrolliert. Da nur Patienten mit einem VAS-Hustenscore $\geq 40 \mathrm{~mm}$ darin waren, bleibt weiterhin offen, ob auch IPF-Patienten mit einer milderen Hustensymptomatik profitieren. Schließlich bleiben aufgrund der recht kurzen Beobachtungszeit die Langzeiteffekte von Pirfenidon auf den IPF-bezogenen Husten unklar. Weitere Studien zu den Pathomechanismen des Hustens bei IPF und dessen Behandlung sind nötig.

\section{Literatur}

1. Ryerson CJ, Abbritti M, Ley B et al. Respirology. 2011;16(6):969-75

2. Horton MR, Santopietro V, Mathew L et al. Ann Intern Med. 2012;157(6):398-406

3. Birring SS, Wijsenbeek MS, Agrawal S et al. Lancet Respir Med. 2017;5(10):806-15

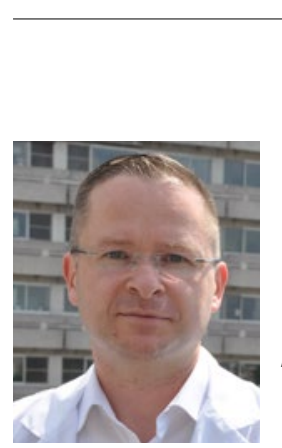

Prof. Dr. med. Philipp Markart

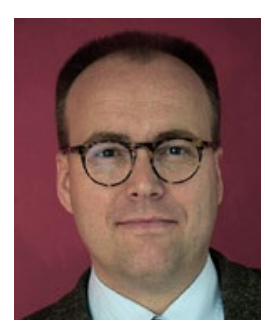

Direktor Medizinische Klinik V, Pneumologie Herz-Thorax-Zentrum Fulda, Klinikum Fulda gAG, Pacelliallee 4, 36043 Fulda med5.sek@klinikum-fulda.de Stellvertretender Leiter Schwerpunkt: "Fibrosierende Lungenerkrankungen", Universitätsklinikum Giessen Marburg GmbH Klinikstrasse 36, 35392 Giessen

Prof. Dr. med. Andreas Günther

Chefarzt AGAPLESION Pneumologische Klinik Waldhof-Elgershausen, 35753 Greifenstein Leiter Schwerpunkt: „Fibrosierende Lungenerkrankungen",

Universitätsklinikum Giessen Marburg GmbH Klinikstrasse 36, 35392 Giessen

Andreas.guenther@innere.med.uni-giessen.de 